\title{
Retrospective analysis of transcatheter arterial chemoembolization treatment for spontaneously ruptured hepatocellular carcinoma
}

\author{
JIXUE ZOU ${ }^{1 *}$, CHAO LI $^{2 *}$, YI CHEN ${ }^{1}$, RONGXIN CHEN ${ }^{1}$, TONGCHUN XUE ${ }^{1}$, \\ XIAOYING XIE ${ }^{1}$, LAN ZHANG $^{1}$ and ZHENGGANG REN ${ }^{1}$ \\ ${ }^{1}$ Liver Cancer Institute, Zhongshan Hospital, Fudan University, Shanghai 200032; ${ }^{2}$ Department of Hepatobiliary Surgery, \\ Eastern Hepatobiliary Surgery Hospital, Second Military Medical University, Shanghai 200433, P.R. China
}

Received May 25, 2019; Accepted September 13, 2019

DOI: $10.3892 / \mathrm{ol} .2019 .11037$

\begin{abstract}
Spontaneous rupture is one of the complications of hepatocellular carcinoma (HCC) associated with a high mortality rate. Transcatheter arterial chemoembolization (TACE) has been widely used in patients with ruptured liver tumors. The aim of the present study was to evaluate the benefits and safety of conventional TACE and the disease prognosis following TACE and surgery with regard to the progression of spontaneously ruptured HCC. The clinical data of 70 patients diagnosed with spontaneous rupture of HCC were retrospectively reviewed. The majority of adverse reactions that occurred following treatment were Grade 2 or below. Grade $3 / 4$ events occurred in 20 patients (14.3\%), which included gastrointestinal hemorrhage, cardiac failure, pulmonary embolism, shock and recurrent tumor rupture. All of these patients recovered and were discharged following symptomatic and supportive treatment, with the exception of two cases of severe hemorrhagic shock and hepatic failure prior to TACE treatment. These patients did not survive during the period of hospitalization. Multivariate analysis identified that a maximum tumor size $>10 \mathrm{~cm}$ and a high serum total bilirubin level $>30 \mu \mathrm{mol} / 1$ were independent factors for determining overall patient survival rate. Additionally, the overall survival rates at 1,6 and 12 months were $92.3,53.8$ and $46.2 \%$ in the TACE group and 100, 87.1 and $54.8 \%$ in the surgery group, respectively. The overall survival rates at 1 and 6 months following TACE were lower than those of the surgery group $(\mathrm{P}<0.05)$. However, the overall survival rates at 12 months were similar $(\mathrm{P}>0.05)$. Patients in the TACE group had a shorter hospital admission compared with those in the resection group
\end{abstract}

Correspondence to: Dr Lan Zhang, Liver Cancer Institute, Zhongshan Hospital, Fudan University, 180 Fenglin Road, Shanghai 200032, P.R. China

E-mail: 13918876432@163.com

${ }^{*}$ Contributed equally

Key words: hepatocellular carcinoma, spontaneous rupture, transarterial chemoembolization, liver resection (median 7 vs. 13 days; $\mathrm{P}<0.01$ ). Therefore, the data demonstrated that conventional TACE therapy was safe and effective for the treatment of spontaneously ruptured HCC. In addition, this type of therapy conferred a similar long-term survival rate with that of open surgery.

\section{Introduction}

Hepatocellular carcinoma (HCC) is one of the most common neoplasms and is considered the third leading cause of cancer-related mortality worldwide (1). Spontaneous rupture with intratumoral hemorrhage is one of the life-threatening complications of HCC. Although the incidence of HCC rupture is $\sim 3-15 \%$, it is associated with high mortality rates that range between 32 and $75 \%$ (2-5). The disease prognosis remains poor in the absence of appropriate treatment in the acute phase (6). Therefore, appropriate treatment selection for patients with $\mathrm{HCC}$ rupture is imperative.

The main treatment for HCC rupture includes emergency or staged hepatectomy and transcatheter arterial chemoembolization (TACE) (7). Several studies have reported a promising outcome following emergency liver resection (8-10). However, patients with advanced HCC tend to have poor hepatic function as a result of chronic hepatitis or liver cirrhosis. Severe cirrhosis or poor patient status, as determined by vital signs and blood examination, often reduce the tolerance of patients to surgery. Conventional TACE has been a widely accepted therapeutic option for unresectable HCC and has shown optimal efficacy (11). TACE is a treatment modality resulting from ischemic necrosis due to arterial embolization. This condition requires the use of cytotoxic drugs and results in a cytotoxic effect (12). In addition to the prevention of tumor progression, TACE is useful in controlling hemorrhage in patients with HCC rupture (13). However, severe complications, including hepatic failure, biliary tract injury, hepatic encephalopathy and renal failure, have been reported in previous studies (14-16). Therefore, assessment of the efficacy and safety of TACE treatment in patients with a ruptured tumor is particularly important. To the best of our knowledge, no definite recommendation has been issued regarding the optimal therapeutic approach in cases of HCC rupture, and the comparative survival benefits of surgery and TACE remain unclear. 
In the present retrospective study, the adverse reactions of TACE and the independent predictors of post-treatment survival outcomes in patients with spontaneously ruptured HCC were investigated. The results were compared with the survival outcomes of TACE and surgery in patients at a single center in China.

\section{Materials and methods}

Patients. The present retrospective study included patients who underwent TACE or surgery for spontaneous HCC rupture between April 2015 and October 2017 at the Department of Hepatic Carcinoma of Fudan University Affiliated Zhongshan Hospital (Shanghai, China). A total of 70 cases with HCC were enrolled. No patient had a recent history of HCC treatment, such as surgery or TACE, within 1 month prior to the diagnosis of HCC rupture. HCC was diagnosed based on the diagnostic criteria issued by the American Association for the Study of Liver Diseases (17). HCC was staged according to the Barcelona Clinic Liver Cancer (BCLC) staging system (18). Spontaneous HCC rupture was diagnosed as disruption of the peritumoral liver capsule with surrounding fluid in the perihepatic region. Detection was performed by dynamic computed tomography (CT) and the diagnosis included the presence of bloody fluid following abdominal puncture.

Database information, such as age, sex and hypertension history, were reviewed. The presence of shock at diagnosis was reviewed and the results of laboratory examinations, including anemia level, baseline liver functions, viral hepatitis findings and $\alpha$-fetoprotein (AFP), were evaluated. Details of HCC tumor status, such as tumor number, tumor size, the presence of vascular thrombosis and the presence of extra-hepatic invasion, were obtained by contrast-enhanced $\mathrm{CT}$ or magnetic resonance imaging (MRI) scans. The present study was approved by the Clinical Research Ethics Committee of Fudan University Affiliated Zhongshan Hospital.

Treatment procedure. Following stabilization of the hemodynamic status by volume replacement and transfusion, the patients underwent a full clinical assessment to evaluate their suitability for surgical treatment. Contraindications for surgical therapy were determined as follows: Presence of poorly controlled predominant ascites and/or hepatic encephalopathy, a prolonged prothrombin time $>4 \mathrm{sec}$ and poor performance status. In hemodynamically unstable patients with an apparent continuous hemorrhage, TACE was considered if reserve liver function was relatively good regardless of the correction for coagulopathy.

TACE or open surgery was immediately performed following diagnosis. These methods were performed concomitantly with supportive treatment. TACE was performed by femoral artery puncture using the Seldinger method (19). A total of 39 patients were enrolled. Prior to TACE, thorough angiography was performed to identify all feeding arteries of the tumor. Digital subtraction angiography was performed to observe tumor staining and to confirm the arterial feeders of the tumor. Following the introduction of a catheter using the Seldinger technique through the femoral artery, a mixture of $5-20 \mathrm{ml}$ lipiodol, epirubicin suspension and gelatin sponge fragments or granules was injected into the tumor-feeding arteries based on the tumor size and the degree of hemorrhage. According to the liver function status, $100-150 \mathrm{mg}$ oxaliplatin or 5-fluorouracil was slowly perfused into the feeding artery under the guidance of digital subtraction angiography by a catheter. For patients with a Child-Pugh score $(20,21)$ of 5 and a white blood cell count $\geq 3 \times 10^{9} / 1,150 \mathrm{mg}$ oxaliplatin was given, while patients with a Child-Pugh score $\geq 6$ or a white blood cell count $<3 \times 10^{9} / 1,100 \mathrm{mg}$ oxaliplatin was given. All patients received $1 \mathrm{~g}$ 5-fluorouracil. Angiography was performed to determine the extent of embolization. A total of 31 patients with ruptured HCC underwent hepatic resection. Hepatic portal interdiction was used in 18 patients and 13 patients received blood transfusion during the procedure.

Follow-up of patients with ruptured HCC following treatment. The median follow-up period was 20 months. During follow-up, the laboratory tests were obtained on the 7th day following the operation. Contrast-enhanced liver CT or MRI scans were performed at 1 month following the operation and subsequently once every 3 months. The main endpoint of the study was the control rate of rupture and hemorrhage of HCC. The overall survival (OS) and the safety of the procedure were used as secondary endpoints.

Statistical analysis. Categorical variables are expressed as frequencies and percentages and were analyzed with the chi-square test. Continuous variables are expressed as medians and ranges and were analyzed with the independent sample t-test. Factors that were found to be significant in univariate analysis were subjected to multivariate analysis. Cox regression hazard model was used to identify independent prognostic factors associated with OS. Propensity score matching (PSM) analysis was used to avoid bias between the TACE and surgery groups. The OS rates were analyzed using the Kaplan-Meier method, and the differences were analyzed with the log-rank test. Statistical analyses were performed using SPSS 21 (IBM Corp.). $\mathrm{P}<0.05$ was considered to indicate a statistically significant difference.

\section{Results}

Baseline characteristics of patients. A total of 70 patients were enrolled in the present study between April 2015 and October 2017. A total of 39 patients were treated with TACE following spontaneous HCC rupture and 31 were treated with open surgery. The median age was 54 years (range, 31-86 years) and the sample size comprised 55 men and 15 women. A total of $52(74.3 \%)$ patients exhibited hepatitis B virus (HBV) infection. Hepatitis $\mathrm{C}$ virus (HCV) infection was not detected in any patient in the present study. The AFP levels in $37(52.9 \%)$ patients were $>400 \mathrm{ng} / \mathrm{ml}$. The Child-Pugh classification indicated that $40(57.1 \%)$ patients were included in class A, 22 (31.4\%) in class B and eight (11.4\%) in class C. BCLC staging at diagnosis indicated that one (1.4\%) patient was classified as stage A, 46 (65.7\%) patients were classified as stage $\mathrm{B}$, and $23(32.9 \%)$ as stage $\mathrm{C}$. Of the 70 patients, 17 (24.3\%) exhibited vascular thrombosis. A total of 40 (57.1\%) patients presented with a single tumor, whereas $30(42.9 \%)$ patients had multiple tumors. The maximum tumor size in $17(24.3 \%)$ and $24(34.3 \%)$ patients was $<5$ and $>10 \mathrm{~cm}$, 
Table I. Clinical characteristics of patients with spontaneously ruptured hepatocellular carcinoma.

\begin{tabular}{|c|c|c|c|c|}
\hline Variable & TACE cases, n (\%) & Surgery cases, n (\%) & P-value & Total cases, $\mathrm{n}(\%)$ \\
\hline Age $>60$ years & $13(33.3)$ & $7(22.6)$ & 0.323 & $20(28.6)$ \\
\hline Hypertension history & $16(41.0)$ & $12(38.7)$ & 0.844 & $28(40.0)$ \\
\hline Child-Pugh & & & $<0.001$ & \\
\hline A & $11(28.2)$ & $29(93.5)$ & & $40(57.1)$ \\
\hline $\mathrm{B}$ & $21(53.8)$ & $1(3.2)$ & & $22(31.4)$ \\
\hline $\mathrm{C}$ & 7 (18) & $1(3.2)$ & & $8(11.4)$ \\
\hline $\mathrm{AFP}>400 \mathrm{ng} / \mathrm{l}$ & $27(69.2)$ & $10(32.3)$ & 0.002 & $37(52.9)$ \\
\hline $\mathrm{ALT}>3 \mathrm{~N}$ & 7 (17.9) & $0(0.0)$ & 0.015 & $7(9.9)$ \\
\hline $\mathrm{AST}>3 \mathrm{~N}$ & $17(43.6)$ & $6(19.4)$ & 0.032 & $23(32.9)$ \\
\hline TBIL $>1.5 \mathrm{~N}$ & $11(28.2)$ & $3(9.7)$ & 0.104 & $14(20.0)$ \\
\hline ALB $\leq 35 \mathrm{~g} / 1$ & $17(43.6)$ & $7(22.6)$ & 0.066 & $24(34.3)$ \\
\hline $\mathrm{PT}>16 \mathrm{sec}$ & $4(10.2)$ & $1(3.2)$ & 0.505 & $5(7.1)$ \\
\hline $\mathrm{Hb}, \mathrm{g} / \mathrm{l}$ & & & $<0.001$ & \\
\hline$>90$ & $13(33.3)$ & $25(80.6)$ & & $36(51.4)$ \\
\hline $90-60$ & $23(59.0)$ & $6(19.4)$ & & $29(41.4)$ \\
\hline$<60$ & $3(7.7)$ & $0(0.0)$ & & $3(4.3)$ \\
\hline Scr $>1.5 \mathrm{~N}$ & $2(5.1)$ & $0(0.0)$ & 0.499 & $2(2.9)$ \\
\hline Positive HBsAg status & $35(89.7)$ & $17(54.8)$ & 0.002 & $52(74.3)$ \\
\hline Maximum tumor size, $\mathrm{cm}$ & & & 0.655 & \\
\hline$\geq 5$ & $9(23.1)$ & $8(25.8)$ & & $17(24.3)$ \\
\hline$>5$ and $\leq 10$ & $18(46.2)$ & $11(35.5)$ & & $29(41.4)$ \\
\hline$>10$ & $12(30.7)$ & $12(38.7)$ & & $24(34.3)$ \\
\hline Tumor number & & & 0.037 & \\
\hline Single & $18(46.2)$ & $22(70.9)$ & & $40(57.1)$ \\
\hline Multiple & $21(53.8)$ & $9(29.0)$ & & $30(42.9)$ \\
\hline Capsule formation & & & 0.208 & \\
\hline Yes & $18(46.2)$ & $19(61.3)$ & & $37(52.9)$ \\
\hline No & $21(53.8)$ & $12(38.7)$ & & $33(47.1)$ \\
\hline Vascular thrombus & & & 0.391 & \\
\hline Yes & $11(28.2)$ & $6(19.4)$ & & $17(24.3)$ \\
\hline No & $28(71.8)$ & $25(80.6)$ & & $53(75.7)$ \\
\hline \multicolumn{5}{|l|}{ Extrahepatic invasion } \\
\hline Yes & $8(20.5)$ & $0(0.0)$ & & $8(11.4)$ \\
\hline No & $31(79.5)$ & $31(100)$ & 0.007 & $62(88.6)$ \\
\hline Shock & $5(12.8)$ & 0 & 0.062 & $5(7.1)$ \\
\hline BCLC stage & & & 0.049 & \\
\hline A & $0(0.0)$ & $1(3.2)$ & & $1(1.4)$ \\
\hline $\mathrm{B}$ & $22(56.4)$ & $24(77.4)$ & & $46(65.7)$ \\
\hline $\mathrm{C}$ & $17(43.6)$ & $6(19.4)$ & & $23(32.9)$ \\
\hline Hospital admission $>10$ days & $9(23.1)$ & $19(61.3)$ & $<0.001$ & $28(40.0)$ \\
\hline
\end{tabular}

TACE, transarterial chemoembolization; AFP, $\alpha$-fetoprotein; N, normal level; ALT, alanine aminotransferase; AST, aspartate aminotransferase; TBIL, total bilirubin; ALB, albumin; PT, prothrombin time; Scr, serum creatinine; HbsAg, hepatitis B surface antigen; BCLC, Barcelona Clinic Liver Cancer.

respectively (Table I). Eight (20.5\%) patients exhibited distant metastasis, whereas three patients presented with metastases in more than one location. Among these, seven metastases were present in the lung, three in the bone and three in distant lymph nodes. These eight patients with distant metastasis were treated with TACE. In the surgery group, no patients exhibited distant metastasis (Table I).

Safety of TACE and surgical treatment. To evaluate the safety of TACE and resection for patients with HCC exhibiting 
Table II. Complications following TACE or surgical treatment.

\begin{tabular}{|c|c|c|c|c|c|c|c|c|c|c|}
\hline \multirow[b]{2}{*}{ Complication } & \multicolumn{2}{|c|}{ I-II degree $(\%)$} & \multicolumn{2}{|c|}{$\geq$ III degree $(\%)$} & \multicolumn{3}{|c|}{ Total (\%) } & \multicolumn{3}{|c|}{$\begin{array}{c}\text { Total after } \\
\text { PSM }(\%) \text { (12 pairs) }\end{array}$} \\
\hline & TACE & Surgery & TACE & Surgery & TACE & Surgery & P-value & TACE & Surgery & P-value \\
\hline Abdominal pain & $15(38.5)$ & $16(51.6)$ & $6(15.4)$ & $12(38.7)$ & $21(53.8)$ & $28(90.3)$ & $<0.001$ & $8(66.7)$ & $9(75.0)$ & 0.653 \\
\hline Nausea and vomiting & $10(25.6)$ & $2(6.5)$ & $2(6.5)$ & 0 & $12(30.8)$ & $2(6.5)$ & 0.012 & $4(33.3)$ & $1(8.3)$ & 0.132 \\
\hline Fever & $14(35.9)$ & $18(58.1)$ & $1(2.6)$ & 0 & $15(38.5)$ & $18(58.1)$ & 0.103 & $5(41.7)$ & $6(50.0)$ & 0.682 \\
\hline Neutrophilia & $15(38.5)$ & $12(38.7)$ & 0 & 0 & $15(38.5)$ & $12(38.7)$ & 0.983 & $4(33.3)$ & $3(25.0)$ & 0.653 \\
\hline Renal failure & $1(2.6)$ & $1(3.2)$ & 0 & 0 & $1(2.6)$ & $1(3.2)$ & 0.869 & 0 & 0 & $\mathrm{NS}$ \\
\hline Cholecystitis & $1(2.6)$ & 0 & 0 & 0 & $1(2.6)$ & 0 & 0.369 & $1(8.3)$ & 0 & 0.307 \\
\hline Recurrent tumor rupture & NS & NS & $2(5.1)$ & $1(3.2)$ & $2(5.1)$ & $1(3.2)$ & 0.696 & 0 & 0 & NS \\
\hline Shock & NS & NS & $1(2.6)$ & $1(3.2)$ & $1(2.6)$ & $1(3.2)$ & 0.869 & 0 & 0 & NS \\
\hline Respiratory failure & 0 & 0 & $1(2.6)$ & 0 & $1(2.6)$ & 0 & 0.369 & 0 & 0 & NS \\
\hline Pulmonary embolism & NS & NS & $1(2.6)$ & 0 & $1(2.6)$ & 0 & 0.369 & 0 & 0 & NS \\
\hline Cardiac failure & 0 & 0 & 0 & $1(3.2)$ & 0 & $1(3.2)$ & 0.259 & 0 & 0 & NS \\
\hline Gastrointestinal hemorrhage & 0 & 0 & 0 & $1(3.2)$ & 0 & $1(3.2)$ & 0.259 & 0 & $1(8.3)$ & 0.307 \\
\hline Hydrothorax & 0 & $2(6.5)$ & 0 & 0 & 0 & $2(6.5)$ & 0.108 & 0 & $1(8.3)$ & 0.307 \\
\hline
\end{tabular}

TACE, transarterial chemoembolization; PSM, propensity score matching; NS not significant.

Table III. Univariate analysis of factors associated with overall survival.

\begin{tabular}{lcc}
\hline Factor & Cases & P-value \\
\hline Hypertension history & 16 & 0.923 \\
Child-Pugh score $>7$ & 14 & 0.094 \\
AFP $>400 \mathrm{ng} / 1$ & 27 & 0.698 \\
ALT $>3 \mathrm{~N}$ & 7 & 0.255 \\
AST $>3 \mathrm{~N}$ & 17 & 0.086 \\
TBIL $>1.5 \mathrm{~N}$ & 11 & 0.001 \\
Maximum tumor size $>10 \mathrm{~cm}$ & 12 & 0.044 \\
Vascular thrombus & 11 & 0.950 \\
Extrahepatic invasion & 8 & 0.622 \\
BCLC stage C & 17 & 0.187 \\
\hline
\end{tabular}

AFP, $\alpha$-fetoprotein; N, normal level; ALT, alanine aminotransferase; AST, aspartate aminotransferase; TBIL, total bilirubin; BCLC, Barcelona Clinic Liver Cancer.

spontaneous tumor rupture, the post-treatment adverse reactions were investigated. Upper abdominal pain (69.0\%), fever $(47.1 \%)$, neutrophilia $(38.6 \%)$ and nausea $(20 \%)$ were the most common adverse reactions. The majority of the toxicity events (54.3\% of all reported events) was Grade 2 or less. Grade 3/4 events occurred in 20 patients (14.3\%), which included gastrointestinal hemorrhage, cardiac failure, pulmonary embolism, shock and recurrent tumor rupture. All of these patients recovered and were discharged following symptomatic and supportive treatment, with the exception of two cases of severe hemorrhagic shock and hepatic failure prior to TACE treatment. These patients did not survive the hospitalization period.
Table IV. Multivariate analysis of factors associated with overall survival.

\begin{tabular}{lccc}
\hline Factor & HR & $95 \%$ CI & P-value \\
\hline TBIL $>30 \mu \mathrm{mol} / 1$ & 0.165 & $0.055-0.497$ & 0.001 \\
Maximum tumor size $>10 \mathrm{~cm}$ & 2.739 & $1.079-6.955$ & 0.034 \\
AST $>3 \mathrm{~N}$ & 0.615 & $0.387-2.176$ & 0.170 \\
Child-Pugh score $>7$ & 1.665 & $0.634-5.878$ & 0.197 \\
\hline
\end{tabular}

HR, hazard ratio; CI, confidence interval; TBIL, total bilirubin; AST, aspartate aminotransferase; $\mathrm{N}$, normal level.

The median duration of hospital admission of patients in the TACE and surgery groups were 7 and 13 days, respectively $(\mathrm{P}<0.01$; Table II).

In the TACE group, 21 patients reported various degrees of upper abdominal pain. This symptom was considered to be associated with tumor rupture following embolism. Nausea and vomiting occurred in 12 patients following TACE treatment. However, the majority of the patients were generally relieved of symptoms within $24-48 \mathrm{~h}$. Fever with a body temperature between 37.5 and $39.5^{\circ} \mathrm{C}$ occurred in 15 patients. This symptom gradually decreased within 3-7 days, possibly due to tumor necrosis and absorption. The majority of the patients exhibited transient liver damage following TACE and recovered to normal or baseline levels within 5-8 days following liver protection therapy. A total of two patients exhibited renal failure, of which only one was newly diagnosed following TACE treatment. Furthermore, one patient developed mild pulmonary embolism and another developed acute cholecystitis of The National Cancer Institute Common Terminology 

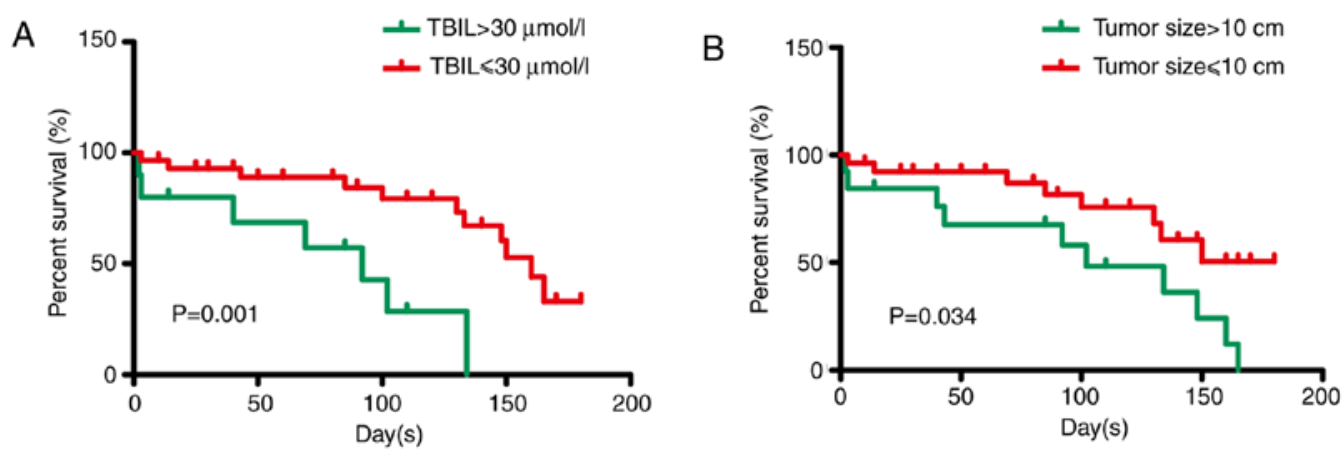

Figure 1. Multivariate analysis of factors related to post-treatment mortality. (A) TBIL $>30 \mu$ mol/1 (P<0.01) and (B) maximum tumor size $>10$ cm (P<0.05) were independent factors determining overall survival. TBIL, total bilirubin.

Criteria for Adverse Events (22) level 2. The cause of this was considered to be ectopic embolism. Following TACE, the patient with cholecystitis recovered within 8 days of antibiotic treatment, intravascular fluid replacement and fasting. A total of six patients presented with shock in the TACE group. However, five of these were in shock prior to the procedure. A total of two patients (5.3\%) exhibited re-rupture of HCC within 1 month following TACE and underwent retreatment. No hepatic encephalopathy, gastrointestinal bleeding, liver abscess or other serious complications occurred.

In the surgery group, a high number of patients presented with abdominal pain and a number of patients exhibited nausea and vomiting following treatment $(\mathrm{P}<0.05)$. There were no significant differences in the incidence of any other complications between these two treatment groups. Two patients exhibited massive hydrothorax, both recovering following thoracic puncturing and piping. One patient experienced gastrointestinal hemorrhage and recovered following 7 days of conservative treatment. One patient presented with grade 3 cardiac failure and shock following resection. This patient finally recovered following symptomatic and supportive treatment.

Univariate and multivariate analysis for predictors of TACE treatment. Prior to multivariate analysis, univariate analysis was conducted in all 39 patients treated with TACE. Univariate analysis resulted in the identification of the following thresholds regarding specific parameters: Maximum tumor size $>10 \mathrm{~cm}(\mathrm{P}=0.044)$, Child-Pugh score $>7(\mathrm{P}=0.094)$, serum total bilirubin levels $>1.5$ times higher than normal levels $(\mathrm{P}=0.001)$ and serum aspartate transferase levels $>3$ times higher than normal levels $(\mathrm{P}=0.086)$ (Table III). These thresholds were used as determinants of poor 30-day survival (Table III). Additional analysis using multivariate Cox regression analysis led to the identification of the following thresholds: Maximum tumor size $>10 \mathrm{~cm}(\mathrm{HR}, 2.739 ; \mathrm{P}=0.034)$ and serum total bilirubin levels $>30 \mu \mathrm{mol} / \mathrm{l}(\mathrm{HR}=0.165, \mathrm{P}=0.001)$. These were used as significant determinants of inverse OS (Table IV; Fig. 1A and B).

Comparison of clinical parameters and survival analysis by treatment types. To determine the efficacy and prognosis of TACE for spontaneously ruptured HCC, the OS rates of the patients with ruptured $\mathrm{HCC}$ who received TACE and/or surgical treatment was examined. Initially, the clinical parameters of the two therapies were analyzed. No significant difference was noted with regard to the parameters of age distribution and sex ratio between the TACE and surgery groups. The history of hypertension and the characteristics of the tumors, such as maximum tumor size, capsule formation and vascular thrombus, were also similar between the two groups (Table I). The total serum bilirubin levels in the TACE group were significantly higher than those in the surgery group $(\mathrm{P}<0.01)$. Patients in the TACE group exhibited higher Child-Pugh scores and BCLC stages compared with those in patients in the surgery group $(\mathrm{P}<0.01$; Fig. 2$)$.

In the TACE group, rupture and hemorrhage of HCC were effectively controlled in 37 (94.9\%) patients. All of these patients were discharged, although four underwent second-stage resection. These four patients were excluded when comparing the OS rates between the two treatments. Two $(5.1 \%)$ patients did not survive during the duration of hospital admission due to severe hemorrhagic shock and consequent hepatic and renal failure. In the surgery group, all patients were effectively treated and discharged. The median survival time periods were 7.4 and 8 months for the TACE and surgery groups, respectively $(\mathrm{P}>0.05)$. The cumulative survival rates at 1, 6 and 12 months were 92.3, 53.8 and 46.2\%, and $100,87.1$ and $54.8 \%$, for the TACE and surgery groups, respectively (Fig. 3). In the TACE group, 17 patients succumbed to hepatic failure, one to brain metastasis and three to massive digestive hemorrhage. The causes of death in the surgery group were similar (12 due to hepatic failure and two due to massive digestive hemorrhage). The cumulative survival rates of the surgery group patients at 1 and 6 months were significantly higher than those noted in the TACE group $(\mathrm{P}<0.01)$. This may be associated with the poor liver function and severe liver cirrhosis noted in the TACE group. However, the mortality rate at the 12-month period did not differ significantly between the two groups ( $\mathrm{P}>0.05$; Fig. 3A). Survival analysis in 12 pairs of patients from the TACE and surgery groups was matched with PSM. The mortality rate at the 12-month period did not differ significantly between the two groups following PSM $(\mathrm{P}>0.05$; Fig. 3B). Additionally, the duration of hospital admission in the TACE group was considerably shorter $(\mathrm{P}<0.01$; Fig. $2 \mathrm{~F})$. These results demonstrated that TACE and surgery treatment resulted in similar OS rates at the 12-month period, although TACE treatment revealed lower OS rates at the 1- and 6-month periods. This indicated that TACE significantly reduces the duration of hospital admission. 

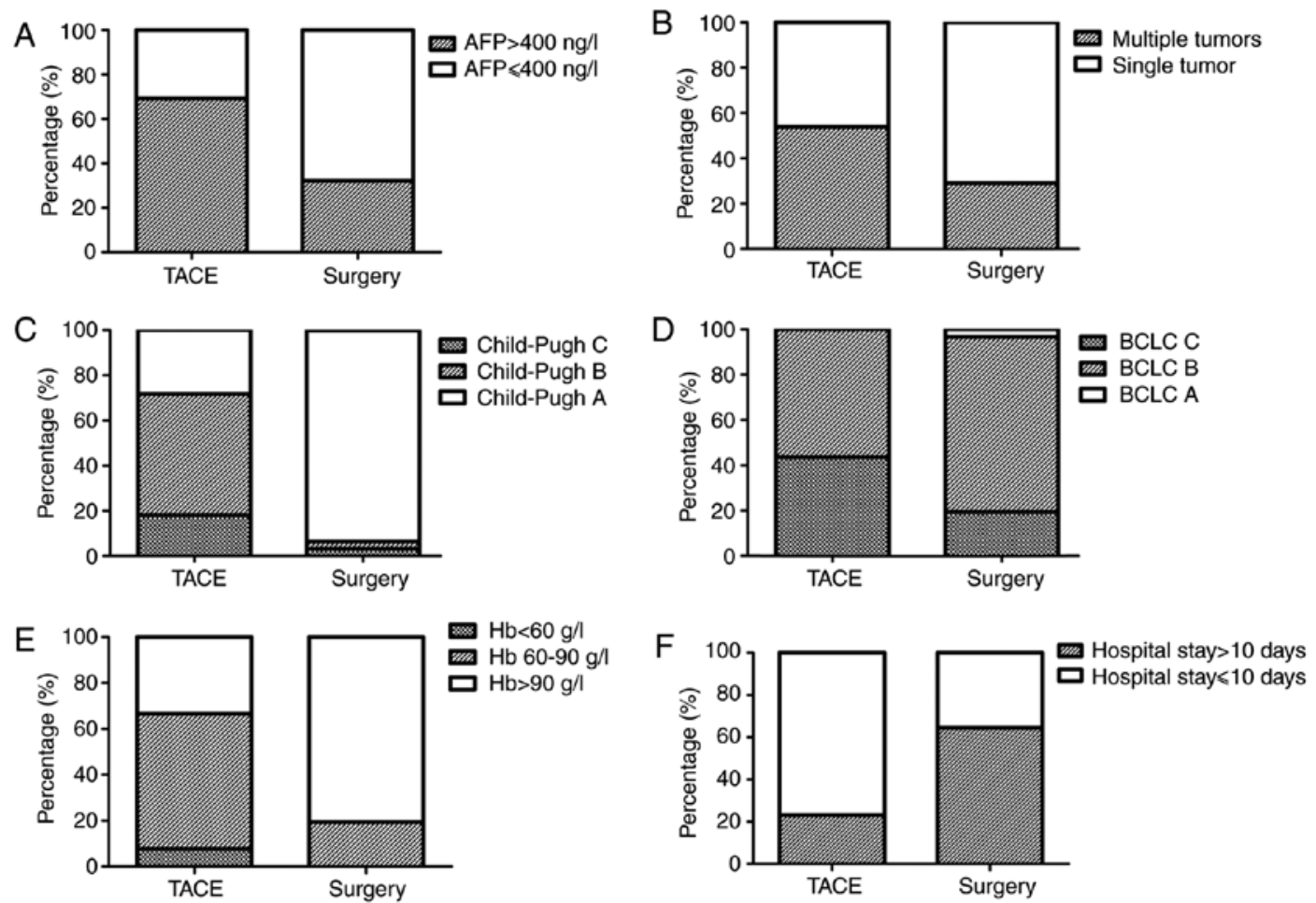

Figure 2. Comparison of clinical parameters and complications in the two treatment groups. (A) AFP levels were significantly lower in the surgical group than in the TACE group $(\mathrm{P}<0.01)$. (B) Single tumor rate was significantly higher in the surgical group than in the TACE group $(\mathrm{P}<0.05)$. (C) Reserve hepatic function (Child-Pugh score) was better in the surgical group than in the TACE group ( $\mathrm{P}<0.01)$. (D) BCLC stage was higher in the surgical group than in the TACE group $(\mathrm{P}<0.01)$. (E) More patients had severe anemia in the TACE group than in the surgery group $(\mathrm{P}<0.05)$. (F) Duration of hospital admission was shorter in the TACE group than in the surgery group $(\mathrm{P}<0.05)$. TACE, transcatheter arterial chemoembolization; AFP, $\alpha$-fetoprotein; BCLC, Barcelona Clinic Liver Cancer; Hb, hemoglobin.
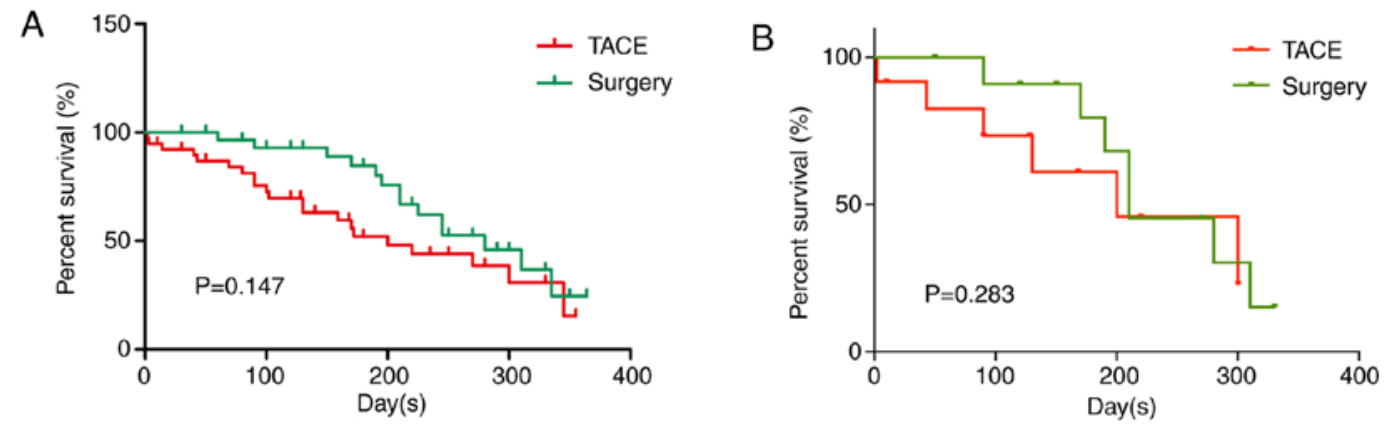

Figure 3. Overall survival of patients with spontaneously ruptured hepatocellular carcinoma following TACE and surgical treatments. The overall survival rates within 12 months in the TACE and surgery groups (A) before and (B) after PSM. The overall survival rate at 12 months did not differ significantly between the two groups $(\mathrm{P}>0.05)$. TACE, transarterial chemoembolization; PSM, propensity score matching.

\section{Discussion}

Spontaneous rupture of HCC is one of the most common emergencies in the advanced stage of this disease (23). Various hypotheses have been formulated to explain the exact mechanism that leads to HCC rupture (24-26). Central necrosis in the growing tumor, portal hypertension, neoplastic invasion causing a local increase of venous pressure within the tumor and a previous vascular injury are associated with the incidence of hemorrhage within the tumor (25). The optimal treatment approach for HCC rupture has not been yet identified (27). Surgery is considered one of the best options for the treatment of HCC with acute spontaneous rupture (28). However, several studies have reported that only a limited number of patients with ruptured HCC can be managed with surgical hepatic resection (29-31). Additionally, surgery is technically difficult to perform in cases of a severely cirrhotic liver (5), and patients with poor liver function and severely ruptured HCC cannot tolerate hepatic resection (32). It has been reported that TACE is effective in achieving immediate hemostasis for ruptured HCC (33). However, the safety and survival benefits of TACE compared with those of surgical resection remain unclear.

The present study demonstrated that the levels of liver enzymes were significantly higher in the first 3 days following TACE compared with those noted at baseline. These differences were reduced within 7 days and the levels of liver 
enzymes returned to normal and/or baseline levels. These results indicated that TACE treatment did not cause severe damage to normal liver tissues. Serious injury to the bile duct is an additional risk of TACE that can lead to cholesteatoma, as reported in previous studies (14). In the present study, only one patient $(2.6 \%)$ presented with this complication and recovered in a few days. Furthermore, no other severe complications, such as hepatic encephalopathy, liver abscess or gastrointestinal bleeding, were noted, which had been reported by previous studies (34). In the present study, the procedure was well tolerated by all study subjects. The general post-procedure complications were minor and self-limiting.

Kirikoshi et al (35) reported that poor liver function only influenced the parameter of short-term mortality rate (21 days), whereas long-term survival (140 days) was not noted in any cases of ruptured HCC. In their study, the size of the tumor (diameter $>7 \mathrm{~cm}$ ) was the only independent factor influencing long-term survival among patients who underwent successful TACE. However, in the present study, the endpoint of a tumor diameter $>5$ or $7 \mathrm{~cm}$ had no significant effect on the survival rate, as determined by multivariate analysis. By contrast, the endpoints of maximum tumor diameter $>10 \mathrm{~cm}$ and serum total bilirubin levels $>30 \mu \mathrm{mol} / 1$ were determined to be independent predictors of survival rate by multivariate analysis. This discrepancy may be associated with the different review time and the different medical history of the subjects. Additional investigations are required to address this discrepancy.

Currently, there is debate regarding the optimal treatment for spontaneous rupture of HCC (23). The 30-day mortality rate of the TACE group was reported to be lower than that of the surgery group in a previous study (36). The present study revealed that the cumulative survival rate in the open surgical group at 1 month was significantly higher than that of the TACE group, which is in accordance with previous studies. However, the cumulative survival rates at 12 months were similar between the two treatment groups. The clinical characteristics between the TACE and surgery groups were also analyzed and the data indicated that patients in the TACE group exhibited higher Child-Pugh scores and BCLC stages compared with those in patients of the surgical group. Furthermore, comparison of the duration of hospital admission revealed that TACE was associated with a significant reduction in the duration of hospital admission compared with that in the surgical group. These results indicated that the long-term survival rate of patients treated with TACE was similar to that of patients treated with open surgery and hospital admission was shorter.

In conclusion, the present study demonstrated that the majority of the patients exhibited transient liver damage following TACE and liver function recovered to normal or baseline levels within a few days of treatment. TACE treatment did not cause serious complications. The maximum tumor size and baseline bilirubin levels were identified as major factors associated with disease prognosis. TACE exhibited acceptable tolerability in patients with spontaneously ruptured $\mathrm{HCC}$, and long-term mortality rates were similar to those in the open surgery treatment group. Furthermore, TACE treatment significantly reduced the duration of hospital admission. Therefore, TACE is considered an option for unresectable HCC rupture due to its efficacy and safety. The present study may provide useful information for clinicians and may aid the determination of the most appropriate treatment option for spontaneous tumor rupture.

\section{Acknowledgements}

Not applicable.

\section{Funding}

The present study was supported by the Young Scientists Fund of the National Natural Science Foundation of China (grant nos. 81702310 and 81502007).

\section{Availability of data and materials}

All data generated and/or analyzed during the present study are included in this published article.

\section{Authors' contributions}

JZ designed the study and wrote the manuscript. CL revised the manuscript, collected and analyzed the data. YC and RC analyzed and interpreted the data. TX and XX statistical analyzed the data. LZ and ZR designed the study. All authors read and approved the final manuscript.

\section{Ethics approval and consent to participate}

The study was approved by the Clinical Research Ethics Committee of Fudan University Affiliated Zhongshan Hospital.

\section{Patient consent for publication}

Not applicable.

\section{Competing interests}

The authors declare that they have no competing interests.

\section{References}

1. Raoul JL and Gilabert M: Hepatocellular carcinoma: Slow progress in a booming epidemic. J Oncol Pract 13: 365-366, 2017.

2. Wang ZM, Lu XS and Qiu F: Hepatectomy for spontaneous rupture of hepatocellular carcinoma without portal triad clamping. Oncol Lett 14: 3997-4004, 2017.

3. Zhu Q, Li J, Yan JJ, Huang L, Wu MC and Yan YQ: Predictors and clinical outcomes for spontaneous rupture of hepatocellular carcinoma. World J Gastroenterol 18: 7302-7307, 2012.

4. Zhao GG, Xu W, Tian YH and Li DX: Survival analysis of patients with spontaneous rupture of hepatocellular carcinoma. Zhonghua Gan Zang Bing Za Zhi 20: 838-842, 2012 (In Chinese).

5. Zhang XF, Wei T, Liu XM and Lv Y: Spontaneous tumor rupture and surgical prognosis of patients with hepatocellular carcinoma. Scand J Gastroenterol 47: 968-974, 2012.

6. Han SR, Kim JM, Choi GS, Park JB, Kwon CH, Kim SJ and Joh JW: Protrusion of hepatocellular carcinoma is a predictor of early recurrence in hepatectomy patients after spontaneous rupture. Ann Surg Treat Res 91: 17-22, 2016.

7. Zhu Q, Qiao GL, Xu C, Guo DL, Tang J, Duan R and Li Y: Partial hepatectomy for spontaneous tumor rupture in patients with hepatocellular carcinoma: A retrospective cohort study. Cancer Manag Res 9: 525-537, 2017. 
8. Ren A, Luo S, Ji L, Yi X, Liang J, Wang J and Wan S: Peritoneal metastasis after emergency hepatectomy and delayed hepatectomy for spontaneous rupture of hepatocellular carcinoma. Asian J Surg 42: 464-469, 2019.

9. Wu JJ,ZhuP,ZhangZG,ZZhang BX, Shu C,Mba'nbo-Koumpa AA Zhang ZW, Huang ZY, Zhang WG, Lau WY and Chen XP: Spontaneous rupture of hepatocellular carcinoma: Optimal timing of partial hepatectomy. Eur J Surg Oncol 45: 1887-1894, 2019.

10. Zhong F, Cheng XS, He K, Sun SB, Zhou J and Chen HM: Treatment outcomes of spontaneous rupture of hepatocellular carcinoma with hemorrhagic shock: A multicenter study. Springerplus 5: 1101, 2016.

11. Hiraoka A, Kumada T, Kudo M, Hirooka M, Koizumi Y, Hiasa Y, Tajiri K, Toyoda H, Tada T, Ochi H, et al: Hepatic function during repeated TACE procedures and prognosis after introducing sorafenib in patients with unresectable hepatocellular carcinoma: Multicenter analysis. Dig Dis 35: 602-610, 2017.

12. Li C, Wen TF, Yan LN, Lu WS, Li B, Wang WT, Xu MQ and Yang JY: Liver resection versus liver resection plus TACE for patients with hepatocellular carcinoma beyond Milan criteria. J Surg Res 209: 8-16, 2017.

13. Maoz D, Sharon E, Chen Y and Grief F: Spontaneous hepatic rupture: 13-year experience of a single center. Eur J Gastroenterol Hepatol 22: 997-1000, 2010.

14. Tu J, Jia Z, Ying X, Zhang D, Li S, Tian F and Jiang G: The incidence and outcome of major complication following conventional TAE/TACE for hepatocellular carcinoma. Medicine (Baltimore) 95: e5606, 2016

15. Zou JH, Zhang L, Ren ZG and Ye SL: Efficacy and safety of cTACE versus DEB-TACE in patients with hepatocellular carcinoma: A meta-analysis. J Dig Dis 17: 510-517, 2016.

16. Hu H, Han XK, Long XR, Fan J, Yan ZP, Wang JH and Liu R Prognostic nomogram for post-surgical treatment with adjuvant TACE in hepatitis B virus-related hepatocellular carcinoma. Oncotarget 7: 58302-58314, 2016.

17. Marrero JA, Kulik LM, Sirlin CB, Zhu AX, Finn RS, Abecassis MM, Roberts LR and Heimbach JK: Diagnosis, staging, and management of hepatocellular carcinoma: 2018 practice guidance by the American Association for the study of liver diseases. Hepatology 68: 723-750, 2018.

18. Gómez-Rodríguez R, Romero-Gutièrrez M, Artaza-Varasa T, González-Frutos C, Ciampi-Dopazo JJ, de-la-Cruz-Pèrez G and Sánchez-Ruano JJ: The value of the Barcelona clinic liver cancer and alpha-fetoprotein in the prognosis of hepatocellular carcinoma. Rev Esp Enferm Dig 104: 298-304, 2012 (In English, Spanish)

19. Driscoll SH, Grollman JH, Ellestedt MH, Po JB, Sample WF and Bos CJ: Single-wall arterial puncture with a disposable needle. Radiology 113: 470, 1974.

20. Child CG and Turcotte JG: Surgery and portal hypertension. Major Probl Clin Surg 1: 1-85, 1964.

21. Pugh RN, Murray-Lyon IM, Dawson JL, Pietroni MC and Williams R: Transection of the oesophagus for bleeding oesophageal varices. Br J Surg 60: 646-649, 1973.

22. Kim AY, Unger K, Wang H and Pishvaian MJ: Incorporating Yttrium-90 trans-arterial radioembolization (TARE) in the treatment of metastatic pancreatic adenocarcioma: A single center experience. BMC Cancer 16: 492, 2016.
23. Rathor M, Lal A and Dhiman RK: Spontaneous rupture of hepatocellular carcinoma. J Clin Exp Hepatol 4: 188-189, 2014

24. You MX, Yu XX, Wu K, Lin YS, Zhu GQ and Shi CS: Analysis of risk factors for spontaneous rupture of hepatocellular carcinoma. Zhonghua Zhong Liu Za Zhi 35: 217-220, 2013 (In Chinese).

25. Zhu LX, Geng XP and Fan ST: Spontaneous rupture of hepatocellular carcinoma and vascular injury. Arch Surg 136: 682-687, 2001

26. Zhu LX, Wang GS and Fan ST: Spontaneous rupture of hepatocellular carcinoma. Br J Surg 83: 602-607, 1996.

27. Wang B, Lu Y, Zhang XF, Yú L, Pan CE and Wu Z: Management of spontaneous rupture of hepatocellular carcinoma. ANZ J Surg 78: 501-503, 2008.

28. Lai EC and Lau WY: Spontaneous rupture of hepatocellular carcinoma: A systematic review. Arch Surg 141: 191-198, 2006.

29. Li J, Huang L, Liu CF, Cao J, Yan JJ, Xu F, Wu MC and Yan YQ Risk factors and surgical outcomes for spontaneous rupture of BCLC stages A and B hepatocellular carcinoma: A case-control study. World J Gastroenterol 20: 9121-9127, 2014.

30. Lee HS, Choi GH, Kang DR, Han KH, Ahn SH, Kim DY, Park JY, Kim SU and Choi JS: Impact of spontaneous hepatocellular carcinoma rupture on recurrence pattern and long-term surgical outcomes after partial hepatectomy. World J Surg 38: 2070-2078, 2014

31. Zhou SJ, Zhang EL, Liang BY, Zhang ZY, Chen XP and Huang ZY: Distilled water lavage during surgery improves long-term outcomes of patients with ruptured hepatocellular carcinoma. J Gastrointest Surg 19: 1262-1270, 2015.

32. Aoki T, Kokudo N, Matsuyama Y, Izumi N, Ichida T, Kudo M, $\mathrm{Ku}$ Y, Sakamoto M, Nakashima O, Matsui O, et al: Prognostic impact of spontaneous tumor rupture in patients with hepatocellular carcinoma: An analysis of 1160 cases from a nationwide survey. Ann Surg 259: 532-542, 2014.

33. Raoul JL, Forner A, Bolondi L, Cheung TT, Kloeckner R and de Baere T: Updated use of TACE for hepatocellular carcinoma treatment: How and when to use it based on clinical evidence. Cancer Treat Rev 72: 28-36, 2019.

34. Hsin IF, Hsu CY, Huang HC, Huang YH, Lin HC, Lee RC, Chiang JH, Lee FY, Huo TI and Lee SD: Liver failure after transarterial chemoembolization for patients with hepatocellular carcinoma and ascites: Incidence, risk factors, and prognostic prediction. J Clin Gastroenterol 45: 556-562, 2011.

35. KirikoshiH,SaitoS, Yoneda M,FujitaK,MawatariH,UchiyamaT, Higurashi T, Imajo K, Sakaguchi T, Atsukawa K, et al: Outcomes and factors influencing survival in cirrhotic cases with spontaneous rupture of hepatocellular carcinoma: A multicenter study. BMC Gastroenterol 9: 29, 2009.

36. Jin YJ, Lee JW, Park SW, Lee JI, Lee DH, Kim YS, Cho SG, Jeon YS, Lee KY and Ahn SI: Survival outcome of patients with spontaneously ruptured hepatocellular carcinoma treated surgically or by transarterial embolization. World J Gastroenterol 19: 4537-4544, 2013.

(i) $($ This work is licensed under a Creative Commons Attribution-NonCommercial-NoDerivatives 4.0 International (CC BY-NC-ND 4.0) License. 